\title{
An analysis of the influence of design parameters on the resonant frequency of PCB coil
}

\author{
Xiuwei Wang ${ }^{1, a}$, Yanping Cong ${ }^{2, b}$ and Haokun Chi ${ }^{3, c}$ \\ ${ }^{1}$ Ocean University of China, No.238, Songling Road, Laoshan District, Qingdao City, Shandong \\ Province, China \\ ${ }^{2}$ Ocean University of China, No.238, Songling Road, Laoshan District, Qingdao City, Shandong \\ Province, China \\ ${ }^{3}$ Ocean University of China, No.238, Songling Road, Laoshan District, Qingdao City, Shandong \\ Province, China \\ a17864270587@sin.cn, bcongyp@ouc.edu.cn, cchihaokun@126.com
}

Keywords: Wireless energy transfer, PCB coil, the resonant frequency, linewidth, edge spacing Abstract. With the development of science and technology, the application of implantable biomedical electronic devices which play an important role in biological research and medical diagnostics becomes increasingly popular[1]. For the implantable electronic equipments, long-term, stable and secure power supply are critical issues. Nowadays wireless energy transfer is one of the most popular research field, it can penetrate the skin to supply electric energy to implantable biomedical electronic devices steady, so it is more and more used in the biomedical field.

The main constraints restrict for wireless charging technology to realize miniaturization and portability is that the volume of the transmission coil is difficult to reduce. The production process of the PCB(printed circuit board) coil is simple, and its width, thickness, line spacing and other parameters can be precisely controlled, mass production of PCB coils have same parameters, meanwhile PCB coil is easy to integrate with external circuit. Compared with the solenoid coil, PCB coil has smaller volume andhigher power density, so it has been widely used in magnetic resonance wireless power transmission field.

In order to improve the efficiency of the radio transmission system fundamentally, by comparing the test methods, we study the PCB coil width and wire spacing, analyze the impact of these two factors on the resonant frequency. And we reach the following conclusion: The resonant frequency is positive correlated to the width of the coil when the wire spacing is fixed. When the coil width is constant, the wire spacing of the coil and the resonant frequency are positively correlated.

This template explains and demonstrates how to prepare your camera-ready paper for Trans Tech Publications. The best is to read these instructions and follow the outline of this text.

\section{Introduction}

Magnetic coupled resonant wireless power transmission technology has the advantages of high transmission distance and high transmission efficiency, and it is a new type of energy transmission technology. Application prospect of the technology is very broad, not only in electric vehicle charging system, wireless sensor network and RFID, get extensive application, operating in household appliances, small mobile tools, traffic tools, industrial robots, oil and mine, water, medical, aerospace and other fields also have great application value and for charging technology and the development of electromagnetic theory also has very important significance[2]. At the same time, one of the frontiers in the field of magnetic coupling resonant wireless power transmission technology is the wireless transmission field, many domestic and foreign scientists are working on high security, high efficiency, long distance, high power wireless energy transmission device[3]. The resonant frequency is a very important index of the magnetic resonance wireless energy transmission technology[4]. However, the theoretical research on the technology is still not perfect, there are still a lack of experimental research, from the overall point of view the technology is still in its infancy, to practical, but also need to solve many problems. If in the process of transmission, encountered 
outside magnetic material will affect the coil inductance changes, resulting in changes in the resonant frequency of the system, that is, the detuning[5], causing the sharp decrease of the transmission efficiency, also working environment temperature change and receive side load changes are likely to lead to detuning. In this paper, the resonance frequency is analyzed from the angle of the change of the transmission performance of the system from the line spacing and line width variation.

Because the wire thickness of the coil is much smaller than the line width, the PCB coil can ignore the influence caused by the proximity effect, and the thickness of the wire is very small. The large AC resistance is the main reason why the transmission efficiency is hard to improve. PCB coils are rectangular, circular and rectangular, and so on. The resonant system has a frequency division in the near distance transmission, so that the efficiency is reduced. In order to solve this problem, this paper analyzes the influence of the line width and the edge spacing on the resonant frequency, and determines the main factors that affect the resonant frequency.

\section{The resonant frequency and its formula}

\section{Resonant frequency}

Resonant frequency is a key condition for the wireless energy transmission with magnetic coupling resonance. When the frequency of the power supply is in line with the natural frequency of the $\mathrm{L}$ and $\mathrm{C}$ circuit, the electromagnetic amplitude of the circuit will reach the peak value, that is, the occurrence of resonance[6]. Resonance, inductance and capacitance equal circuit is purely resistive, and minimum impedance. At this point, the magnetic field in the circuit inductance can be exchanged with the electric field in the capacitor[7]. When the receiving coil of the transmission device is in agreement with the resonant frequency of the sending coil, most of the energy of the transmitting coil can be absorbed by the receiving circuit, and the purpose of the magnetic coupling resonant wireless energy transmission is achieved.

\section{Resonant frequency formula}

Below, this paper combines the RLC parallel circuit as shown in Figure 1, to calculate the resonant frequency of the circuit.

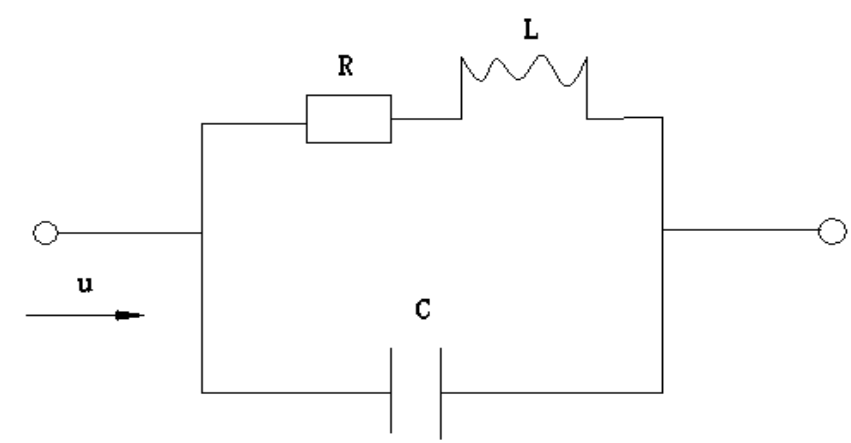

Figure 1 R-L-C parallel circuit

A power supply voltage of the R-L-C parallel circuit is provided:

$$
\mathrm{u}=\sqrt{2} U \sin \omega t
$$

When the circuit is in resonance, $\omega C-\frac{\omega L}{R^{2}+(\omega L)^{2}}=0$, we have the conclusion that:

$$
\omega=\omega_{0}=\frac{1}{\sqrt{L C}} \sqrt{1-\frac{C R^{2}}{L}}
$$

When $R<\sqrt{\frac{L}{C}}$, that is $\frac{C R^{2}}{L}<1$, At this time the resonant frequency is $\omega_{\circ} \approx \frac{1}{2 \pi \sqrt{L C}}$. 


\section{Coil characteristics influence}

The coil characteristic has very important influence to the radio energy transmission. The choice of the wire and the coil is various, the appropriate coil can obtain the higher resonant frequency, increase the energy density, reduce the skin effect. In the literature, a new type of PCB patch type induction coil is proposed, which can reduce the volume of the coil, increase the energy density, and effectively reduce the skin effect[8]. This paper introduces the method of reducing the skin effect by using the Liz line or the silver plated wire[9]. The Liz line can make the electric current and electromagnetic field distribute evenly, the surface resistance of the silver plated wire is small, and the skin depth is small[10]. The structure of the coil includes the wire diameter and the electrical conductivity, the diameter of the coil, and the winding way. Coil winding way a lot, there are rectangular, circular ring, spiral, etc.. Taking rectangular coil as an example, figure 2 and figure 3 are the coil winding way.

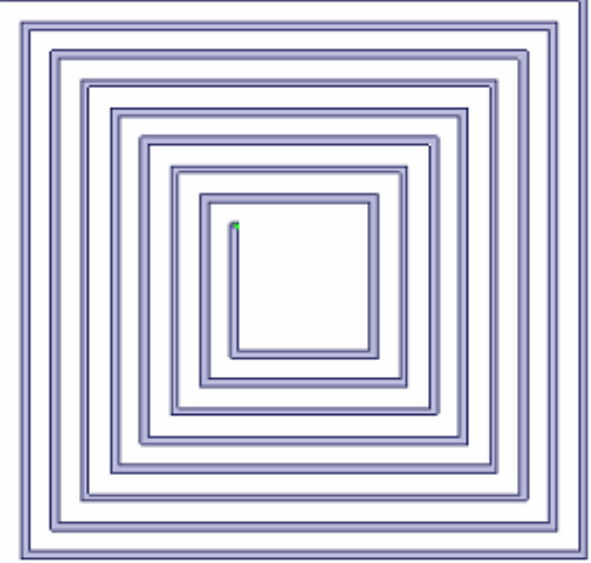

Figure 2 Flat rectangular coil winding way

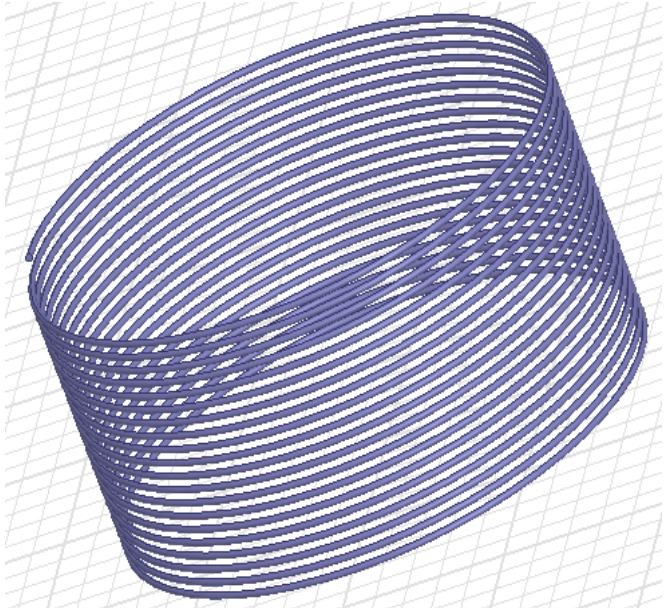

Figure 3 Three dimensional spiral coil winding

In this paper, the PCB coil material is copper, PCB board material is FR4_epoxy, and the physical parameters of the coil are represented by the following symbols: l is the length of the coil, $\mathrm{w}$ represents the width of copper, s represents the distance between adjacent coils margin line , $\mathrm{h}$-c represents copper thickness (In this paper, the thickness of copper is all $0.035 \mathrm{~mm}$ ), $\mathrm{h} \_\mathrm{f}$ represents the thickness of the substrate FR4_epoxy (In this paper, the thickness of PCB plate is all 0.33mm), $\mathrm{n}$ represents the total number of turns of the coil.

Table 1 PCB parameter setting of rectangular coil

\begin{tabular}{c|c}
\hline Electrical parameter & Set value \\
\hline $\mathrm{n}$ & 7 turns \\
$\mathrm{s}$ & $10-60 \mathrm{mil}$ \\
$\mathrm{l}$ & $4 \mathrm{~cm}$ \\
w & $5-50 \mathrm{mil}$ \\
h_f & $0.33 \mathrm{~mm}$ \\
h_c & $0.035 \mathrm{~mm}$ \\
\hline
\end{tabular}

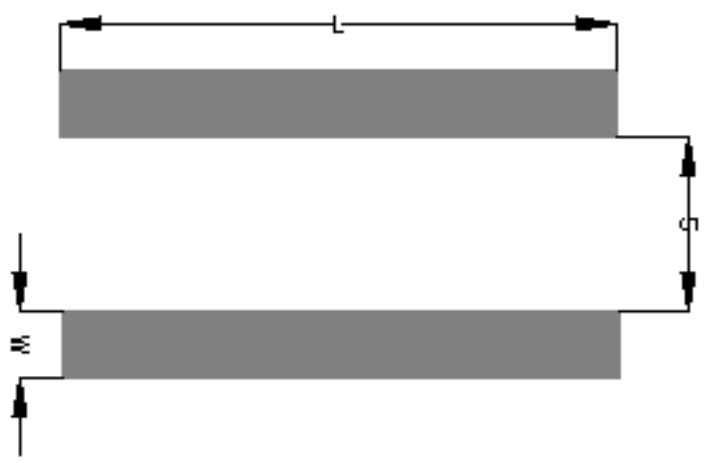

Figure 4 The width of the coil and the line spacing 


\section{Side coil spacing and width parameters}

Through the simulation analysis, this paper has obtained the coil side spacing and the coil width contrast data, and the simulation of the data of the list analysis, draw the conclusion that the influence of the edge distance and line width on the resonant frequency.

Table 2 Effects of different line width and edge spacing on the resonance frequency

\begin{tabular}{cllllll}
$\begin{array}{r}\text { ry(mil) } \\
\text { S(mil) }\end{array}$ & 10 & 20 & 30 & 40 & 50 & 60 \\
\hline 5 & 49.8 & 62.16 & 73.82 & 85.08 & 98.54 & 112.1 \\
10 & 52.13 & 64.56 & 76.2 & 90.4 & 103.66 & 117.7 \\
15 & 58.3 & 67.66 & 81.22 & 98.4 & 109.52 & 123.78 \\
20 & 66.9 & 75.52 & 89.9 & 104.56 & 119.38 & 135.1 \\
25 & 75.2 & 80.62 & 96.52 & 105.42 & 120.24 & 143.84 \\
30 & 80 & 88.2 & 96.1 & 117 & 132.9 & 149.78 \\
35 & 89.42 & 93.86 & 106.9 & 116.16 & 142.48 & 157.8 \\
40 & 92.85 & 100.12 & 113.06 & 129.46 & 147.26 & 165.4 \\
45 & 101.71 & 104 & 109.8 & 136.04 & 155.12 & 172.92 \\
50 & 106.23 & 110.86 & 129.08 & 145.52 & 163.16 & 181.4 \\
\hline
\end{tabular}

According to table 2 data can be seen, when the linewidth is certain and edge spacing changes from 10mil to 60mil, the resonant frequency of the coil increases with the increase of edge spacing, that is,the resonant frequency is positive correlated to edge spacing; When the coil edge spacing is certain, linewidth from $5 \mathrm{mil}$ increased to 50mil, the resonant frequency of the coil increases with the increase of the linewidth, namely resonance frequency and linewidth into positive correlation.

\section{PCB rectangular coil parameters influence on the resonant frequency}

\section{PCB rectangular coil of wire effects on the resonant frequency of side space}

Through HFSS simulation software to the rectangular coil were several sets of simulation, and the simulation data is analysed and processed, Figure 5 edge spacing effect on the resonant frequency curves are obtained. The abscissa is edge spacing $\mathrm{S}$, ordinate is resonant frequency $\mathrm{F}$, set different edge spacing $S_{i}$ (where $\mathrm{i}=1,2,3 \ldots .$. ), Corresponding to different resonant frequencies are $F_{\mathrm{i}}$ (wherein $\mathrm{i}=1,2,3 \ldots$...), and the resonance frequency of the series resonant circuit is $f_{r}$, according to the resonant circuit and made analysis, it made the following formula:

$$
\begin{aligned}
& f_{1}=f_{r}-\frac{\mathrm{S}_{i} \bullet F_{i}}{2} \\
& f_{2}=f_{r}+\frac{S_{i} \bullet F_{i}}{2}
\end{aligned}
$$

Among them, $f 1, f 2$ are the corresponding resonant frequency of the different edge distance, which is further derived from the above formula, the relationship between the line spacing and the resonant frequency is obtained:

$$
f_{r}=\frac{2 \bullet S_{i}}{\sqrt{f_{1} \bullet f_{2}}}
$$

It can be known that the gap between the coil and the resonant frequency is positively correlated, that is, when the line width is certain, the increase of the side space of the coil will lead to the increase of the resonance frequency. 


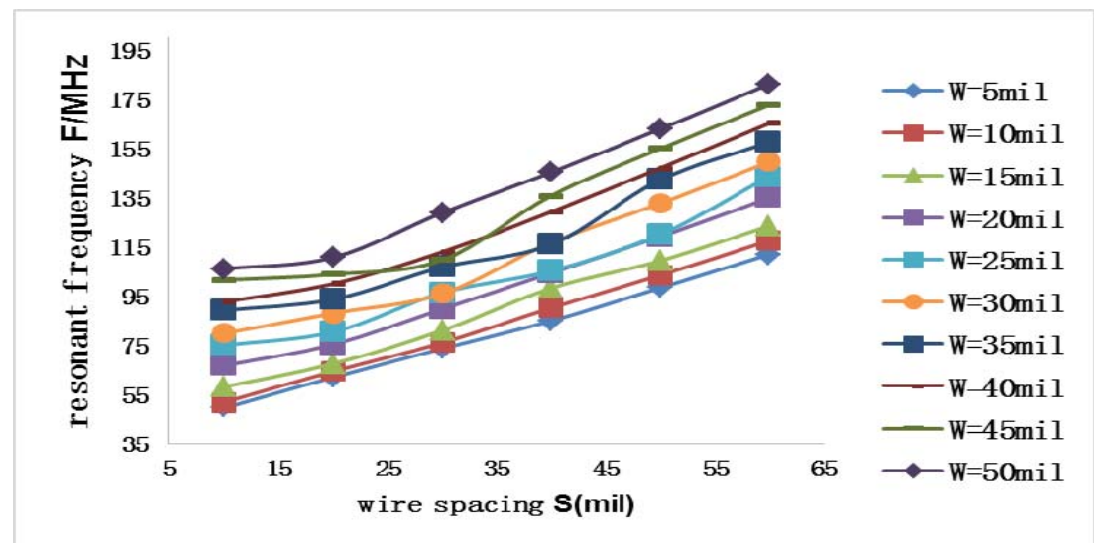

Figure 5 Influence of edge spacing on the resonance frequency of the coil

But with respect to the individual parameters, the increase in the side space of the coil leads to the increase of the resonance frequency, which is not so obvious. Conductor width can not increase too much, because the width of the wire will affect the distribution of magnetic field across the surface of the PCB board, therefore, the wire side spacing is one of the important factors that affect the resonant frequency.

\section{Effect of PCB rectangular coil resonant frequency linewidth}

Similarly, the HFSS simulation software to the rectangular coil did several sets of simulation, and the simulation data were analysis, Figure 6 linewidth influence on resonant frequency curves are obtained. where the horizontal axis is the line width $\mathrm{W}$, the vertical axis is the resonant frequency $\mathrm{F}$, where any two set different width ${ }^{W_{i}}$ corresponding to the resonance frequencies are $f_{i}, f_{j}$. according to the model of the resonant circuit, the resonant energy transfer system in the following conclusions:

$$
f_{r}=\frac{1}{2 \pi \sqrt{L C}}
$$

Where $f_{r}$ is the resonant frequency of the coil, $\mathrm{L}$ and $\mathrm{C}$ are the inductance of the coil and capacitor. In Figure 7, when a certain time line spacing, the effects of different width of the resonance frequency is not so obvious.Rate of change of the resonance frequency is defined as: ${ }=\frac{\left|f_{i}-f_{j}\right|}{w_{i}}$,After validation of analytical results:

When $\eta \leq 0.05 \%$, Rate of change of the resonance frequency $\eta$ tends to 0 , then finds the resonant frequency is not changed. That is when the spacing of the coil is constant, with the increase in width of the resonance frequency initially increase slightly, after the rate of change due to $\eta=\frac{\left|f_{i}-f_{j}\right|}{w_{i}}>0.05 \%$.

So at this time rate of change $\eta$ of is greater than the rate of change of edge spacing $30 \mathrm{mil}$ when that side of the pitch with the increase of the rate of change is gradually increased.

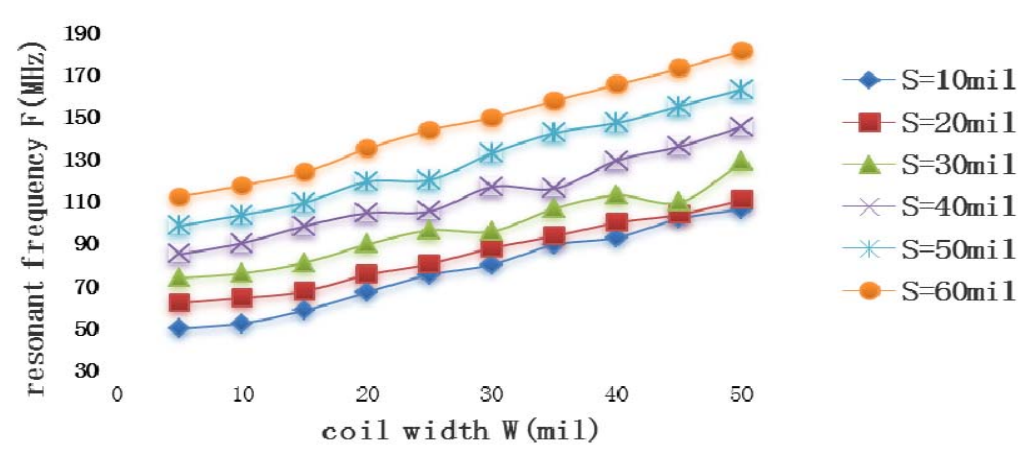

Figure 6 Effect of linewidth on the resonance frequency of the coil 


\section{Conclusions}

In this paper, the radio transmission technology is briefly reviewed, and the calculation formula of resonant frequency is introduced, especially the influence of the electrical parameters of the coil section on the resonant frequency is analyzed in detail. The design of the coil is one of the key technologies of radio energy transmission. The high resonant frequency of the coil has great influence on the transmission performance of the system, including transmission distance, transmission efficiency, transmission power and so on. The effects of different resonant frequency on the efficiency of different linewidth and edge spacing are analyzed. Such as the coil parameters of the design, including the coil shape, turn, turn spacing,linewidth , material selection, etc., this paper on the use of HFSS simulation of the coil is also briefly described. Finally, we analyzed coil width and space of turns and influence on the resonant frequency, and drew the conclusion: The resonant frequency is positive correlated to the width of the coil when the wire spacing is fixed. When the coil width is constant, the wire spacing of the coil and the resonant frequency are positively correlated.

\section{Acknowledgements}

This paper received funding of China International Scientific and Technological Cooperation Special (2013DFA10490) and Qingdao innovation and entrepreneurship leading talent project (13-cx-2).

\section{References}

[1] Manolatou C, Khan M J, Fan Shanhui, et al. Coupling of modes analysis of resonant channel add-drop filters[J]. IEEE Journal of Quantum Electronics, 1999,35(9): 1322-1331

[2] Hirai J J, Kim T W, Kawamura A. Wireless transmission of power and information for cableless linear motor drive[J]. IEEE Transactions on Power Electronics, 2000, 15(1): 21-27.

[3] Andre Kurs, Aristeidis Karalis, Robert Moffatt, et al.Wireless power transfer via strongly coupled magnetic resonances[J]. Science, 2007, 317(6): 83-86.

[4] Li H L, Hu A P, Covic G A, et al. Optimal coupling condition of IPT sysytem for activeing maximum power transfer[J]. Electronics Letters, 2009, 45(1):76-77.

[5] M Ali, G R Dougal. A miniature packaged rectenna for wireless power transmission and date telemetry[C]. IEEE International Workshop on Antenna Technology Small Antennas and Novel Metamaterials, Mar. 2006:225-228.

[6] Chwei-Sen Wang, Oskar H Stielau. Design considerations for a contactless electric vehicle battery charger[C]. IEEE Transactions on Industrial Electronics, 2005,52(5): 1308-1314.

[7] Darrin J Yong. Wireless powering and date telementry for biomedical implants[C]. The 31st Annual international Conference of the IEEE EMBS,Minnesota, USA, 2009: 3221-3224.

[8] Joaquin J Casanova, Zhen NingLow, Jenshan Lin.Design and optimization of a class-E amplifier for a loosely coupled plannar wireless power system[C].IEEE Transactions on Circuits and Systems, 2009,56(11): 830-834.

[9] Qingxin Yang, Haiyan Chen, Suzhen Liu, et al. Dynamic modeling of magnetic system constructedwith giant magneto strictive thin film using elementfree galerkin method[J]. IEEE Transactions on Magnetics, 2006, 42(4): 939-942.

[10] Marracci M, Tellini B,Zappacosta C,elal. Critical parame ters for mutual inductance between Rogowski coil and primary conductor[J]. IEEE Transactions on Instrumentation and Measurement,2011,60(2):625-632. 